\title{
THE DELETERIOUS EFFECT IN DOGS OF A DRY PROTEIN RATION ${ }^{1}$
}

\author{
By T. S. DANOWSKI, J. R. ELKINTON, AND A. W. WINKLER
}

(From the Department of Internal Medicine, Yale University School of Medicine, New Haven)

(Received for publication March 27, 1944)

The present study seeks to determine whether dry protein without additional water increases dehydration. It was undertaken in a search for information whereby the survival time of individuals with inadequate supplies of water could be prolonged. Dogs deprived of food only, or of all intake, served as controls. The experimental variants included dry protein, dry carbohydrate, mixtures of dry protein and carbohydrate, fresh fillets of fish, and 20 per cent urea solution. Balances of water, electrolytes, and nitrogen were determined and the physiological reactions of the animals observed.

\section{MATERIAL AND METHODS}

Adult dogs were used throughout. Fourteen animals were fasted without water as controls. Data from 8 of these experiments have already been published (1 to 3 ). Data from the other 6 , including 4 rehydration periods, are included in Tables IA and IB. Five other animals were fasted but allowed water as desired. Eight animals received a thoroughly dried proprietary dog food, "SAS," in various amounts, enclosed in large gelatin capsules. One lot of dog food contained by analysis: 431 m.eq. of sodium, 275 m.eq. of chloride, 100 m.eq. of potassium, and 76.7 grams of nitrogen per kilogram. One kilogram of another lot contained the same amount of sodium and potassium, 292 m.eq. of chloride, and 73.8 grams of nitrogen. Both contained 9 per cent of fat, 10 per cent of bone, traces of carbohydrate, inorganic salts, and vitamin concentrates. This dog food constitutes under ordinary circumstances a complete maintenance ration. Empty capsules averaged 0.9 grams in weight and contained 152 grams of nitrogen per kilogram of capsule. Six to 18 capsules filled with dry ration and lubricated with mineral oil were swallowed after insertion into the hypopharynx. Vomiting was observed in only a few instances, contrary to the experience of Straub (4). Five dogs were fed carbohydrate and protein in the form of glucose enclosed in gelatin capsules without water. Three animals were given carbohydrate alone in the form of granulated sugar or of nitrogen free candy balls. Two dogs were fed fillets of raw haddock. By analysis of combined aliquots from 12 of the fish fillets, each kilogram

1 The authors are indebted to Dr. Samuel Harvey for the use of the Surgical Laboratory, and to Mrs. Theodore Mintz for technical assistance. contained: water 808 grams, sodium 29.8 m.eq., chloride 26.7 m.eq., potassium 87.3 m.eq., and nitrogen 28.4 grams.

All stool and urine specimens were analyzed for total nitrogen, potassium, sodium, and chloride. A small loss of ammonia from stools may have occurred during drying. Body weight, and the concentration of non-protein nitrogen in blood and of chloride and protein in serum were determined at intervals. In some experiments, analyses of serum for sodium and potassium were included. The

TABLE IA

Control experiments: fasting with and without water Body weight, blood and serum analyses

\begin{tabular}{|c|c|c|c|c|c|c|c|}
\hline \multirow{2}{*}{ Exp.* } & \multirow{2}{*}{ Time } & \multirow{2}{*}{$\underset{\text { weight }}{\text { Body }}$} & \multirow{2}{*}{$\begin{array}{l}\text { Blood } \\
\text { NPN }\end{array}$} & \multicolumn{4}{|c|}{ Serum } \\
\hline & & & & $\begin{array}{c}\text { Total } \\
\text { protein }\end{array}$ & $\mathrm{Na}$ & $\mathrm{Cl}$ & $\mathbf{K}$ \\
\hline & day & kgm. & $\begin{array}{c}\text { per } \\
\text { cent }\end{array}$ & $\begin{array}{c}\text { grams } \\
\text { per } \\
\text { cent }\end{array}$ & $\begin{array}{c}\text { m.eq. } \\
\text { per } \\
\text { liter }\end{array}$ & $\begin{array}{c}\text { m.eq. } \\
\text { per } \\
\text { liter }\end{array}$ & $\begin{array}{c}\text { m.eq. } \\
\text { per } \\
\text { liter }\end{array}$ \\
\hline $23 \mathrm{C}$ & $\begin{array}{l}0 \\
7\end{array}$ & $\begin{array}{l}9.94 \\
8.12\end{array}$ & $\begin{array}{l}29 \\
35\end{array}$ & $\begin{array}{l}6.00 \\
6.98\end{array}$ & $\begin{array}{l}140.1 \\
146.5\end{array}$ & $\begin{array}{l}102.9 \\
109.0\end{array}$ & $\begin{array}{l}4.78 \\
4.63\end{array}$ \\
\hline $30 \mathrm{~B}$ & $\begin{array}{r}0 \\
10\end{array}$ & $\begin{array}{l}7.54 \\
6.00\end{array}$ & $\begin{array}{l}24 \\
31\end{array}$ & $\begin{array}{l}5.59 \\
6.69\end{array}$ & $\begin{array}{l}142.5 \\
151.6\end{array}$ & $\begin{array}{l}107.2 \\
113.0\end{array}$ & $\begin{array}{l}4.99 \\
5.38\end{array}$ \\
\hline $32 B$ & $\begin{array}{l}0 \\
7\end{array}$ & $\begin{array}{l}7.26 \\
6.12\end{array}$ & $\begin{array}{l}21 \\
40\end{array}$ & $\begin{array}{l}5.60 \\
6.81\end{array}$ & $\begin{array}{l}142.6 \\
146.3\end{array}$ & $\begin{array}{l}106.5 \\
110.6\end{array}$ & $\begin{array}{l}3.99 \\
5.37\end{array}$ \\
\hline $35 \mathrm{~A}$ & $\begin{array}{r}0 \\
10\end{array}$ & $\begin{array}{l}7.30 \\
5.86\end{array}$ & $\begin{array}{l}26 \\
29\end{array}$ & $\begin{array}{l}5.88 \\
6.53\end{array}$ & $\begin{array}{l}140.0 \\
149.3\end{array}$ & $\begin{array}{l}107.5 \\
114.4\end{array}$ & $\begin{array}{l}4.67 \\
5.07\end{array}$ \\
\hline $35 \mathrm{D}$ & $\begin{array}{r}0 \\
15 \\
16 \\
\end{array}$ & $\begin{array}{l}7.44 \\
5.36 \\
5.68 \\
\end{array}$ & $\begin{array}{l}23 \\
28 \\
20 \\
\end{array}$ & $\begin{array}{l}7.12 \\
6.29 \\
5.12 \\
\end{array}$ & \begin{tabular}{|l|}
137.2 \\
153.3 \\
127.1 \\
\end{tabular} & $\begin{array}{r}106.1 \\
121.3 \\
97.8 \\
\end{array}$ & $\begin{array}{l}3.28 \\
4.69 \\
4.83 \\
\end{array}$ \\
\hline $37 \mathrm{C}$ & $\begin{array}{r}0 \\
15 \\
16 \\
\end{array}$ & $\begin{array}{l}7.56 \\
5.50 \\
5.78\end{array}$ & $\begin{array}{l}26 \\
20 \\
18\end{array}$ & $\begin{array}{l}7.12 \\
8.02 \\
6.51\end{array}$ & $\begin{array}{l}141.5 \\
158.5 \\
127.7\end{array}$ & $\begin{array}{r}105.4 \\
120.9 \\
97.5\end{array}$ & $\begin{array}{l}3.53 \\
4.74 \\
4.85\end{array}$ \\
\hline $23 \mathrm{D}$ & $\begin{array}{l}0 \\
8\end{array}$ & $\begin{array}{l}9.76 \\
8.26\end{array}$ & $\begin{array}{l}27 \\
27\end{array}$ & $\begin{array}{l}5.69 \\
6.07\end{array}$ & \begin{tabular}{|l|}
145.3 \\
146.1 \\
\end{tabular} & $\begin{array}{l}107.4 \\
110.7\end{array}$ & $\begin{array}{l}4.73 \\
5.00\end{array}$ \\
\hline $32 \mathrm{C}$ & $\begin{array}{l}0 \\
8\end{array}$ & $\begin{array}{l}7.38 \\
6.22\end{array}$ & $\begin{array}{l}29 \\
18\end{array}$ & $\begin{array}{l}5.29 \\
5.65\end{array}$ & \begin{tabular}{|l|}
142.9 \\
145.2
\end{tabular} & $\begin{array}{l}110.4 \\
107.4\end{array}$ & $\begin{array}{l}4.47 \\
5.30\end{array}$ \\
\hline $30 \mathrm{C}$ & $\begin{array}{r}0 \\
14\end{array}$ & $\begin{array}{l}7.06 \\
5.52\end{array}$ & $\begin{array}{l}22 \\
30\end{array}$ & $\begin{array}{l}6.40 \\
5.60\end{array}$ & $\begin{array}{l}149.5 \\
146.9\end{array}$ & $\begin{array}{l}109.2 \\
108.0\end{array}$ & $\begin{array}{l}5.26 \\
5.74\end{array}$ \\
\hline $35 \mathrm{C}$ & $\begin{array}{r}0 \\
15\end{array}$ & $\begin{array}{l}8.80 \\
6.48\end{array}$ & $\begin{array}{l}32 \\
31\end{array}$ & $\begin{array}{l}6.40 \\
5.55\end{array}$ & $\begin{array}{l}148.5 \\
141.0\end{array}$ & $\begin{array}{l}104.6 \\
109.7 \\
\end{array}$ & $\begin{array}{l}4.93 \\
4.38 \\
\end{array}$ \\
\hline $37 \mathrm{~B}$ & $\begin{array}{r}0 \\
15\end{array}$ & $\begin{array}{l}8.73 \\
6.62\end{array}$ & $\begin{array}{l}22 \\
30\end{array}$ & $\begin{array}{l}7.33 \\
6.33\end{array}$ & $\begin{array}{l}143.5 \\
138.5\end{array}$ & $\begin{array}{l}109.7 \\
104.4\end{array}$ & $\begin{array}{l}5.74 \\
5.08\end{array}$ \\
\hline
\end{tabular}
IB.

* Water withheld or administered as indicated in Table 
TABLE IB

Control experiments: fasting with and without water

Balances of electrolytes and nitrogen; calculation of insensible loss of weight (IL) and of changes in total water balance $(\Delta \mathrm{W})$, in extracellular fluid $(\Delta \mathrm{E})$, and in intracellular fluid $(\Delta \mathrm{I})$.

\begin{tabular}{|c|c|c|c|c|c|c|c|c|c|c|c|}
\hline \multirow{2}{*}{ Exp. } & \multirow{2}{*}{$\begin{array}{l}\text { Period } \\
\text { from } \\
\text { start }\end{array}$} & \multirow{2}{*}{ Water } & \multirow{2}{*}{ Urine } & \multicolumn{4}{|c|}{ Balance } & \multirow{2}{*}{ IL } & \multirow{2}{*}{$\Delta \mathrm{W}$} & \multirow{2}{*}{$\Delta \mathbf{E}$} & \multirow{2}{*}{$\Delta \mathrm{III}^{*}$} \\
\hline & & & & $\mathrm{Na}$ & $\mathrm{Cl}$ & $\mathbf{K}$ & $\mathbf{N}$ & & & & \\
\hline $23 \mathrm{C}$ & $\begin{array}{l}\text { days } \\
0 \text { to } 7\end{array}$ & $\begin{array}{l}c c . \\
0\end{array}$ & $\begin{array}{l}c c . \\
430\end{array}$ & $\begin{array}{c}\text { m.eq. } \\
-56.6\end{array}$ & $\begin{array}{c}\text { m.eq. } \\
-81.8\end{array}$ & $\begin{array}{c}\text { m.eq. } \\
-75.4\end{array}$ & $\begin{array}{l}\text { grams } \\
-15.3\end{array}$ & $\begin{array}{l}\mathrm{kgm} . \\
1.28\end{array}$ & $\begin{array}{l}\text { liters } \\
-1.35\end{array}$ & $\begin{array}{l}\text { liters } \\
-0.83\end{array}$ & $\begin{array}{l}\text { liters } \\
-0.68\end{array}$ \\
\hline $30 \mathrm{~B}$ & 0 to 10 & 0 & 175 & -39.2 & -37.3 & -77.6 & -18.7 & 1.26 & -1.10 & -0.41 & -0.69 \\
\hline $32 \mathrm{~B}$ & 0 to 7 & 0 & 235 & -28.0 & -31.2 & -44.8 & -8.3 & 0.89 & -0.88 & -0.31 & -0.39 \\
\hline $35 \mathrm{~A}$ & 0 to 10 & 0 & 205 & -64.8 & -33.8 & -64.2 & -14.1 & 1.15 & -1.04 & -0.33 & -0.62 \\
\hline $35 \mathrm{D}$ & $\begin{array}{r}0 \text { to } 15 \\
15 \text { to } 16\end{array}$ & $\begin{array}{r}0 \\
1450\end{array}$ & $\begin{array}{l}520 \\
715\end{array}$ & $\begin{array}{l}-31.4 \\
-8.9\end{array}$ & $\begin{array}{l}-46.3 \\
-12.7\end{array}$ & $\begin{array}{l}-68.2 \\
-22.2\end{array}$ & $\begin{array}{r}-22.6 \\
-2.7\end{array}$ & $\begin{array}{l}1.28 \\
0.35\end{array}$ & $\begin{array}{r}-1.47 \\
+0.42\end{array}$ & $\begin{array}{l}-0.55 \\
+0.21\end{array}$ & $\begin{array}{l}-0.73 \\
+0.29\end{array}$ \\
\hline $37 \mathrm{C}$ & $\begin{array}{r}0 \text { to } 15 \\
15 \text { to } 16\end{array}$ & $\begin{array}{r}0 \\
730\end{array}$ & $\begin{array}{l}553 \\
170\end{array}$ & $\begin{array}{l}-62.7 \\
-11.2\end{array}$ & $\begin{array}{l}-37.5 \\
-14.2\end{array}$ & $\begin{array}{r}-75.0 \\
-9.4\end{array}$ & $\begin{array}{l}-23.6 \\
-3.0\end{array}$ & $\begin{array}{l}1.28 \\
0.23\end{array}$ & $\begin{array}{l}-1.41 \\
+0.36\end{array}$ & $\begin{array}{l}-0.52 \\
+0.23\end{array}$ & $\begin{array}{l}-0.80 \\
+0.44\end{array}$ \\
\hline $23 \mathrm{~F}$ & $\begin{array}{l}0 \text { to } 3 \\
3 \text { to } 4\end{array}$ & $\begin{array}{r}0 \\
590\end{array}$ & $\begin{array}{l}150 \\
280\end{array}$ & & $\begin{array}{r}-22.0 \\
-5.0\end{array}$ & $\begin{array}{r}-25.0 \\
-5.9\end{array}$ & $\begin{array}{l}-6.2 \\
-\quad 3.0\end{array}$ & $\begin{array}{l}0.58 \\
0.29\end{array}$ & $\begin{array}{l}-0.59 \\
-0.08\end{array}$ & $\begin{array}{r}-0.28 \\
+0.14\end{array}$ & $\begin{array}{l}-0.14 \\
+0.05\end{array}$ \\
\hline $32 \mathrm{E}$ & $\begin{array}{l}0 \text { to } 3 \\
3 \text { to } 4\end{array}$ & $\begin{array}{r}0 \\
310\end{array}$ & $\begin{array}{l}138 \\
220\end{array}$ & & $\begin{array}{r}-7.0 \\
-4.7\end{array}$ & $\begin{array}{l}-21.2 \\
-2.0\end{array}$ & $\begin{array}{l}-4.9 \\
-2.6\end{array}$ & $\begin{array}{l}0.28 \\
0.08\end{array}$ & $\begin{array}{l}-0.34 \\
-0.07\end{array}$ & $\begin{array}{r}-0.09 \\
0.00\end{array}$ & $\begin{array}{l}-0.21 \\
+0.11\end{array}$ \\
\hline $23 \mathrm{D}$ & 0 to 8 & 1125 & 720 & & -51.2 & -73.1 & -16.6 & 1.81 & -1.06 & -0.49 & -0.50 \\
\hline $32 \mathrm{C}$ & 0 to 8 & 2330 & 2050 & & -29.6 & -55.4 & -12.6 & 1.18 & -0.74 & -0.21 & -0.42 \\
\hline $30 \mathrm{C}$ & 0 to 14 & 815 & 595 & -44.0 & -42.3 & -69.4 & -23.6 & 1.70 & -0.99 & -0.32 & -0.42 \\
\hline $35 \mathrm{C}$ & 0 to 15 & 1870 & 1480 & -63.0 & -50.1 & -92.8 & -23.9 & 2.30 & -1.60 & -0.49 & -0.41 \\
\hline $37 \mathrm{~B}$ & 0 to 15 & 1500 & 1210 & -67.5 & -63.1 & -94.8 & -29.1 & 2.17 & -1.51 & -0.32 & -0.42 \\
\hline
\end{tabular}

* $\Delta \mathrm{I}_{\mathrm{I}}$ omitted, since it is simply $\Delta \mathrm{W}-\Delta \mathrm{E}$.

water content of serum was calculated from the total protein concentration by the formula: $\mathrm{W}_{\mathrm{s}}=99.30-0.889 \mathrm{P}$. This formula was derived from simultaneous determinations of total protein concentration and water content of 54 samples of dog serum in previous experiments. Changes in total body water, in extracellular fluid volume, and intracellular fluid volume were calculated by methods previously described (1). Extracellular fluid changes were based on chloride balances.

\section{RESULTS}

Data on control animals deprived of food and, in some instances, of water as well, are presented in Tables IA and IB. Data from the animals which received various foodstuffs are recorded in Tables IIA and IIB. In Figures 1 and 2, the amount of protein metabolized daily is correlated with the survival time and with the daily water loss through various channels.

(A) Period of survival. There is a rough inverse proportion between the amount of protein metabolized daily and the period of survival. All but 2 of the control animals were resuscitated after 10 to 20 days of complete water deprivation. Survival was shortest in the animals with the highest daily metabolism of protein. The protein metabolized was not directly proportional to the protein ingested, but did increase with it.

(B) Rate of water loss. (Figure 2.) The average daily rate of water loss was much more rapid in the protein-fed dogs than in the controls. If the 2 dogs which received the smallest daily allotment of protein are excluded, the mean daily balance of water in the 6 other dogs was $-29.1 \pm 4.3 \mathrm{cc}$. per kilogram per day. The difference between this and the control value of $-16.6 \pm 3.2$ is statistically highly significant. This increased rate of total water loss in the protein-fed dogs resulted from a larger daily urine volume. An increase in the daily insensible loss of water as a result of increased metabolism 
may have been a contributory factor. The daily urine volume was practically doubled in the protein-fed dogs. This was associated with a higher daily metabolism of protein necessitating a greater excretion of nitrogen. It is evident that dry protein does spare body protein but it accelerates dehydration.

Animals fed carbohydrate alone, or together with the small quantity of protein of the gelatin capsules, resembled, on the whole, the controls in the amount of nitrogen metabolized, the volume of urine, and the total loss of water. In Dog 23, represented in Figure 1 by the cross farthest to the left, the carbohydrate definitely spared body protein, decreased urine nitrogen, and diminished dehydration. Experiments with this same animal deprived of all intake, deprived of food alone (23C and 23D, Tables IA and IB),

TABLE IIA

Ingestion of various foodstuffs without supplementary water Body weight, blood and serum analyses

\begin{tabular}{|c|c|c|c|c|c|c|c|}
\hline \multirow{2}{*}{ Exp.* } & \multirow{2}{*}{ Period } & \multirow{2}{*}{$\begin{array}{c}\text { Body } \\
\text { weight }\end{array}$} & \multirow{2}{*}{$\begin{array}{l}\text { Blood } \\
\text { NPN }\end{array}$} & \multicolumn{4}{|c|}{ Serum } \\
\hline & & & & $\begin{array}{c}\text { Total } \\
\text { protein }\end{array}$ & $\mathrm{Na}$ & $\mathrm{Cl}$ & $\mathbf{K}$ \\
\hline $12 \mathrm{D}$ & $\begin{array}{r}d a y \\
0 \\
14\end{array}$ & $\begin{array}{r}\text { kgm. } \\
12.20 \\
9.31\end{array}$ & \begin{tabular}{|c} 
mgm. \\
per cent \\
25 \\
105
\end{tabular} & \begin{tabular}{|c|} 
grams \\
per cent \\
6.65 \\
7.55 \\
\end{tabular} & $\begin{array}{c}\begin{array}{c}\text { m. eq. } \\
\text { per liter } \\
144.5 \\
188.8\end{array} \\
\end{array}$ & 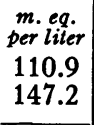 & $\begin{array}{c}\begin{array}{c}\text { m. eq. } \\
\text { per liter } \\
3.33 \\
5.40\end{array} \\
\end{array}$ \\
\hline $13 \mathrm{D}$ & $\begin{array}{r}0 \\
8 \\
13\end{array}$ & $\begin{array}{r}11.20 \\
9.08 \\
8.11\end{array}$ & $\begin{array}{r}29 \\
28 \\
130\end{array}$ & $\begin{array}{l}6.29 \\
7.67 \\
9.65\end{array}$ & \begin{tabular}{|l|}
141.9 \\
162.7 \\
190.3
\end{tabular} & $\begin{array}{l}109.3 \\
129.3 \\
158.3\end{array}$ & $\begin{array}{l}4.49 \\
5.32 \\
6.08\end{array}$ \\
\hline $23 \mathrm{G}$ & $\begin{array}{r}0 \\
3 \\
10 \\
13\end{array}$ & $\begin{array}{r}11.46 \\
10.06 \\
8.41 \\
9.52\end{array}$ & $\begin{array}{l}35 \\
35 \\
51 \\
24\end{array}$ & $\begin{array}{l}6.62 \\
7.58 \\
8.38 \\
5.37\end{array}$ & $\begin{array}{l}144.9 \\
152.2 \\
188.5 \\
144.0\end{array}$ & $\begin{array}{l}105.1 \\
109.1 \\
146.6 \\
110.0\end{array}$ & $\begin{array}{l}5.37 \\
4.52 \\
4.18 \\
4.36\end{array}$ \\
\hline $32 F$ & $\begin{array}{l}0 \\
3 \\
5\end{array}$ & $\begin{array}{l}7.38 \\
6.52 \\
5.89\end{array}$ & $\begin{array}{l}31 \\
48 \\
98\end{array}$ & $\begin{array}{l}6.49 \\
7.76\end{array}$ & $\begin{array}{l}149.8 \\
172.1\end{array}$ & $\begin{array}{l}113.1 \\
133.9\end{array}$ & $\begin{array}{l}4.94 \\
4.47\end{array}$ \\
\hline 39 & $\begin{array}{l}0 \\
7\end{array}$ & $\begin{array}{r}11.08 \\
8.76\end{array}$ & $\begin{array}{l}30 \\
98\end{array}$ & $\begin{array}{l}6.60 \\
8.07\end{array}$ & & $\begin{array}{l}104.8 \\
160.6\end{array}$ & \\
\hline 38 & $\begin{array}{l}0 \\
7\end{array}$ & $\begin{array}{r}11.22 \\
8.36\end{array}$ & $\begin{array}{l}31 \\
63\end{array}$ & $\begin{array}{l}6.69 \\
6.93\end{array}$ & & $\begin{array}{l}101.9 \\
145.3\end{array}$ & \\
\hline 41 & $\begin{array}{l}0 \\
9\end{array}$ & $\begin{array}{l}6.90 \\
5.12 \\
\end{array}$ & $\begin{array}{l}32 \\
38\end{array}$ & $\begin{array}{l}5.80 \\
6.96\end{array}$ & & $\begin{array}{l}109.9 \\
144.1\end{array}$ & \\
\hline 40 & $\begin{array}{l}0 \\
7\end{array}$ & $\begin{array}{l}6.42 \\
5.17\end{array}$ & $\begin{array}{l}27 \\
99\end{array}$ & $\begin{array}{l}7.13 \\
8.89\end{array}$ & & $\begin{array}{l}104.0 \\
149.8\end{array}$ & \\
\hline $23 \mathrm{E}$ & $\begin{array}{l}0 \\
6\end{array}$ & $\begin{array}{l}6.63 \\
5.00 \\
\end{array}$ & $\begin{array}{l}28 \\
37\end{array}$ & $\begin{array}{l}6.58 \\
7.63 \\
\end{array}$ & \begin{tabular}{|l|}
146.1 \\
150.8 \\
\end{tabular} & $\begin{array}{r}99.3 \\
107.8 \\
\end{array}$ & $\begin{array}{l}4.47 \\
5.10 \\
\end{array}$ \\
\hline $32 \mathrm{D}$ & $\begin{array}{l}0 \\
6\end{array}$ & $\begin{array}{l}4.37 \\
3.80\end{array}$ & $\begin{array}{l}23 \\
39\end{array}$ & $\begin{array}{l}6.76 \\
6.50\end{array}$ & \begin{tabular}{|l|}
146.2 \\
148.3
\end{tabular} & $\begin{array}{l}105.5 \\
106.5\end{array}$ & $\begin{array}{l}4.73 \\
5.15\end{array}$ \\
\hline
\end{tabular}

TABLE IIA-Continued

\begin{tabular}{|c|c|c|c|c|c|c|c|}
\hline \multirow{2}{*}{ Exp.* } & \multirow{2}{*}{ Period } & \multirow{2}{*}{$\begin{array}{c}\text { Body } \\
\text { weight }\end{array}$} & \multirow{2}{*}{$\begin{array}{l}\text { Blood } \\
\text { NPN }\end{array}$} & \multicolumn{4}{|c|}{ Serum } \\
\hline & & & & $\begin{array}{c}\text { Total } \\
\text { protein }\end{array}$ & $\mathrm{Na}$ & $\mathrm{Cl}$ & $\mathbf{K}$ \\
\hline $23 \mathrm{H}$ & $\begin{array}{r}d a y \\
0 \\
11 \\
28\end{array}$ & $\begin{array}{r}\text { kgm. } \\
10.10 \\
9.70 \\
9.22\end{array}$ & \begin{tabular}{|c}
$\underset{\text { mgm. }}{\text { per cent }}$ \\
24 \\
26 \\
28
\end{tabular} & $\begin{array}{c}\text { grams } \\
\text { per cent } \\
6.35 \\
6.66 \\
6.44\end{array}$ & 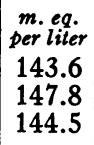 & 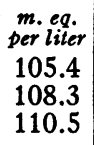 & 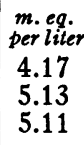 \\
\hline $37 \mathrm{~A}$ & $\begin{array}{r}0 \\
12 \\
28\end{array}$ & $\begin{array}{l}7.92 \\
7.16 \\
6.72\end{array}$ & $\begin{array}{l}23 \\
25 \\
28\end{array}$ & $\begin{array}{l}5.73 \\
6.72 \\
7.12\end{array}$ & \begin{tabular}{|l}
146.5 \\
145.0 \\
148.0
\end{tabular} & $\begin{array}{l}105.7 \\
108.4 \\
103.9\end{array}$ & $\begin{array}{l}4.36 \\
4.01 \\
4.96\end{array}$ \\
\hline $30 \mathrm{~A}$ & $\begin{array}{l}0 \\
8\end{array}$ & $\begin{array}{l}7.62 \\
6.34\end{array}$ & $\begin{array}{l}33 \\
32\end{array}$ & $\begin{array}{l}5.84 \\
6.48\end{array}$ & $\begin{array}{l}149.4 \\
147.5\end{array}$ & $\begin{array}{l}106.3 \\
111.1\end{array}$ & \begin{tabular}{|l|}
4.62 \\
5.09
\end{tabular} \\
\hline 31 & $\begin{array}{l}0 \\
7\end{array}$ & $\begin{array}{l}6.76 \\
5.51\end{array}$ & $\begin{array}{l}27 \\
25\end{array}$ & $\begin{array}{l}5.06 \\
7.17\end{array}$ & $\begin{array}{l}145.9 \\
167.0\end{array}$ & $\begin{array}{l}108.8 \\
126.6\end{array}$ & $\begin{array}{l}4.67 \\
5.97\end{array}$ \\
\hline $35 \mathrm{~B}$ & $\begin{array}{l}0 \\
8\end{array}$ & $\begin{array}{l}6.96 \\
5.74\end{array}$ & $\begin{array}{l}31 \\
32\end{array}$ & $\begin{array}{l}6.41 \\
6.21\end{array}$ & $\begin{array}{l}147.5 \\
150.7\end{array}$ & $\begin{array}{l}102.6 \\
114.6\end{array}$ & $\begin{array}{l}5.20 \\
4.41\end{array}$ \\
\hline $23 \mathrm{~L}$ & $\begin{array}{l}0 \\
9\end{array}$ & $\begin{array}{r}11.45 \\
9.37\end{array}$ & $\begin{array}{l}30 \\
22\end{array}$ & $\begin{array}{l}6.04 \\
7.26\end{array}$ & $\begin{array}{l}149.5 \\
160.0\end{array}$ & $\begin{array}{l}105.2 \\
113.9\end{array}$ & $\begin{array}{l}5.21 \\
3.96\end{array}$ \\
\hline $37 \mathrm{G}$ & $\begin{array}{l}0 \\
9\end{array}$ & $\begin{array}{l}8.90 \\
6.88\end{array}$ & $\begin{array}{l}30 \\
24\end{array}$ & $\begin{array}{l}6.05 \\
8.36\end{array}$ & $\begin{array}{l}144.9 \\
160.3\end{array}$ & $\begin{array}{l}109.3 \\
118.4\end{array}$ & $\begin{array}{l}5.08 \\
5.27\end{array}$ \\
\hline $23 \mathrm{M}$ & $\begin{array}{l}0 \\
9\end{array}$ & $\begin{array}{r}10.11 \\
8.86\end{array}$ & $\begin{array}{l}22 \\
22\end{array}$ & $\begin{array}{l}6.08 \\
6.56\end{array}$ & $\begin{array}{l}145.4 \\
151.6\end{array}$ & $\begin{array}{l}105.3 \\
116.9\end{array}$ & \\
\hline $47 \mathrm{~A}$ & $\begin{array}{l}0 \\
6\end{array}$ & $\begin{array}{l}6.76 \\
5.79\end{array}$ & $\begin{array}{l}24 \\
31\end{array}$ & $\begin{array}{l}4.96 \\
4.95\end{array}$ & $\begin{array}{l}142.8 \\
153.4\end{array}$ & $\begin{array}{l}105.8 \\
118.0\end{array}$ & \\
\hline $49 \mathrm{~A}$ & $\begin{array}{l}0 \\
9\end{array}$ & $\begin{array}{l}16.29 \\
13.80\end{array}$ & $\begin{array}{l}29 \\
38\end{array}$ & $\begin{array}{l}6.09 \\
7.34\end{array}$ & $\begin{array}{l}148.8 \\
160.3\end{array}$ & $\begin{array}{l}113.7 \\
115.2\end{array}$ & \\
\hline
\end{tabular}

* Ingestion of dry protein alone in experiments $12 \mathrm{D}$ through 40; of urea in saline in 23E; of urea in 32D; of raw fish in $23 \mathrm{H}$ and $37 \mathrm{~A}$; of protein and carbohydrate in $30 \mathrm{~A}$ through $37 \mathrm{G}$; and of carbohydrate alone in $23 \mathrm{M}$ through $49 \mathrm{~A}$.

given protein alone, and given carbohydrate with protein (23G and 23L, Tables IIA and IIB), indicate clearly that an economy of body water resulted from the administration of carbohydrate. It is probable that carbohydrate produced a similar effect in the other animals, but that the change was not large enough to place the animals outside the range of the control groups. It was not possible to represent this graphically, since these points overlay those of the control group.

(C) Renal response. In general, the urine volumes and the concentrations of nitrogen in the urine were greater in the dogs fed protein than in the controls. Experiment $32 \mathrm{D}$ is the sole exception. This dog had the largest urine volume and the shortest survival, despite a very low concentration of nitrogen in the urine. It is 
TABLE IIB

Ingestion of various foodstuffs without supplementary water

Balances of electrolytes and nitrogen; calculation of insensible loss of weight (IL) and of changes in total water balance $(\Delta W)$, in extracellular fluid $(\Delta E)$, and in intracellular fluid $(\Delta I)$.

\begin{tabular}{|c|c|c|c|c|c|c|c|c|c|c|c|c|c|}
\hline \multirow[b]{2}{*}{ Expt. } & \multirow[b]{2}{*}{ Period } & \multirow[b]{2}{*}{ Intake } & \multicolumn{3}{|c|}{ Nitrogen } & \multirow[b]{2}{*}{$\begin{array}{l}\text { Urine } \\
\text { vol. }\end{array}$} & \multicolumn{3}{|c|}{ Balance } & \multirow[b]{2}{*}{ IL } & \multirow[b]{2}{*}{$\Delta \mathrm{W}$} & \multirow[b]{2}{*}{$\Delta \mathbf{E}$} & \multirow[b]{2}{*}{$\Delta \mathrm{I}_{\mathbf{n}} * *$} \\
\hline & & & $\underset{\text { In- }}{\text { In- }}$ & $\begin{array}{c}\text { Ex- } \\
\text { creted } \\
\text { in } \\
\text { urine }\end{array}$ & $\begin{array}{c}\text { Ex- } \\
\text { creted } \\
\text { in } \\
\text { stool }\end{array}$ & & $\mathbf{N a}$ & $\mathbf{C l}$ & $\mathbf{K}$ & & & & \\
\hline $12 \mathrm{D}$ & $\begin{array}{l}\text { days } \\
0 \text { to } 14\end{array}$ & $\begin{array}{c}\text { grams } \\
\text { Dry protein }\end{array}$ & $\begin{array}{r}\text { grams } \\
36.6\end{array}$ & $\begin{array}{r}\text { grams } \\
37.0\end{array}$ & $\begin{array}{c}\text { grams } \\
5.1\end{array}$ & $\begin{array}{l}c c . \\
435\end{array}$ & $\begin{array}{l}\text { m.eq. } \\
+77.8\end{array}$ & $\begin{array}{l}m . e q . \\
+26.1\end{array}$ & $\left|\begin{array}{c}m . e q . \\
-6.06\end{array}\right|$ & $\begin{array}{l}\mathrm{kgm} . \\
2.59\end{array}$ & $\begin{array}{l}\text { liters } \\
-2.36\end{array}$ & $\begin{array}{c}\text { liters } \\
-0.61\end{array}$ & $\begin{array}{l}\text { liters } \\
-1.46\end{array}$ \\
\hline $13 \mathrm{D}$ & $\begin{array}{l}0 \text { to } 8 \\
8 \text { to } 13\end{array}$ & $\begin{array}{l}\text { Dry protein } \\
\text { Dry protein }\end{array}$ & $\begin{array}{l}20.8 \\
13.0\end{array}$ & $\begin{array}{l}30.7 \\
15.9\end{array}$ & $\begin{array}{l}1.9 \\
2.3\end{array}$ & $\begin{array}{l}680 \\
265\end{array}$ & $\begin{array}{l}-34.3 \\
+50.2\end{array}$ & $\begin{array}{l}-38.3 \\
+\quad 25.3\end{array}$ & $\begin{array}{l}-80.0 \\
-17.0\end{array}$ & $\begin{array}{l}1.58 \\
0.71\end{array}$ & $\begin{array}{l}-1.84 \\
-0.80\end{array}$ & $\begin{array}{l}-0.72 \\
-0.28\end{array}$ & $\begin{array}{l}-1.07 \\
-0.66\end{array}$ \\
\hline $23 G$ & $\begin{array}{c}0 \text { to } 3 \\
3 \text { to } 10 \\
10 \text { to } 13\end{array}$ & $\begin{array}{l}\text { Dry protein } \\
\text { Dry protein } \\
\text { Dry protein }\end{array}$ & $\begin{array}{l}16.4 \\
37.1 \\
15.9\end{array}$ & $\begin{array}{l}19.0 \\
45.2 \\
17.4\end{array}$ & $\begin{array}{l}8.0 \\
3.5\end{array}$ & $\begin{array}{l}405 \\
670 \\
440\end{array}$ & $\begin{array}{l}-51.7 \\
+61.0 \\
+21.1\end{array}$ & $\begin{array}{r}-69.5 \\
+\quad 4.5 \\
+\quad 10.1\end{array}$ & $\begin{array}{r}-35.7 \\
-58.4 \\
+\quad 1.6\end{array}$ & $\begin{array}{l}0.99 \\
1.06 \\
0.71\end{array}$ & $\begin{array}{l}-1.15 \\
-1.50 \\
+1.32\end{array}$ & $\begin{array}{r}-0.69 \\
-0.54 \\
+0.68\end{array}$ & $\begin{array}{l}-0.43 \\
-1.11 \\
+0.96\end{array}$ \\
\hline $32 F$ & $\begin{array}{l}0 \text { to } 3 \\
3 \text { to } 5\end{array}$ & $\begin{array}{l}\text { Dry protein } \\
\text { Dry protein }\end{array}$ & $\begin{array}{r}16.1 \\
8.8\end{array}$ & $\begin{array}{r}15.1 \\
3.6\end{array}$ & 3.7 & $\begin{array}{l}455 \\
120\end{array}$ & -9.8 & -20.3 & -37.8 & $\begin{array}{l}0.46 \\
0.46\end{array}$ & $\begin{array}{l}-0.81 \\
-0.48\end{array}$ & -0.43 & -0.57 \\
\hline 39 & 0 to 7 & Dry protein & 62.3 & 44.2 & 6.5 & 925 & & +5.0 & & 1.55 & -2.13 & -0.95 & \\
\hline 38 & 0 to 7 & Dry protein & 75.7 & 59.3 & 9.8 & 1135 & & -65.0 & . & 1.86 & -2.56 & -1.24 & \\
\hline 41 & 0 to 9 & Dry protein & 59.6 & 38.3 & 9.6 & 620 & & +17.0 & & 1.31 & -1.62 & -0.32 & \\
\hline 40 & 0 to 7 & Dry protein & 51.5 & 35.2 & 6.6 & 550 & & +29.0 & & 0.97 & -1.28 & -0.34 & \\
\hline $23 \mathrm{E}$ & 0 to 6 & $\begin{array}{l}20 \text { per cent urea in } \\
0.9 \text { per cent } \mathrm{NaCl}\end{array}$ & 13.8 & 25.4 & & 740 & & -64.7 & -82.4 & 1.38 & -1.63 & -0.76 & -0.83 \\
\hline $32 \mathrm{D}$ & 0 to 6 & 0.9 per cent $\mathrm{NaCl}$ & 0 & 8.6 & & 280 & & -15.6 & -44.4 & 0.62 & -0.57 & -0.14 & -0.34 \\
\hline $23 \mathrm{H}$ & $\begin{array}{r}0 \text { to } 11 \\
11 \text { to } 28\end{array}$ & $\begin{array}{l}\text { Raw fish } \\
\text { Raw fish }\end{array}$ & $\begin{array}{l}133.7 \\
206.6\end{array}$ & $\begin{array}{l}127.4 \\
188.0\end{array}$ & & $\begin{array}{l}2450 \\
3100\end{array}$ & $\begin{array}{l}+3.0 \\
-14.4\end{array}$ & $\begin{array}{r}+\quad 4.7 \\
+\quad 33.0\end{array}$ & $\begin{array}{l}+28.8 \\
+42.0\end{array}$ & $\begin{array}{l}2.29 \\
4.11\end{array}$ & $\begin{array}{l}-0.56 \\
-0.61\end{array}$ & $\begin{array}{r}-0.04 \\
+0.21\end{array}$ & $\begin{array}{l}+0.06 \\
+0.19\end{array}$ \\
\hline $37 \mathrm{~A}$ & $\begin{array}{r}0 \text { to } 12 \\
12 \text { to } 28\end{array}$ & $\begin{array}{l}\text { Raw fish } \\
\text { Raw fish }\end{array}$ & $\begin{array}{l}59.3 \\
97.2\end{array}$ & $\begin{array}{l}45.6 \\
78.1\end{array}$ & & $\begin{array}{l}1100 \\
1650\end{array}$ & $\begin{array}{l}-12.0 \\
+6.1\end{array}$ & $\begin{array}{l}-10.9 \\
+16.6\end{array}$ & $\begin{array}{l}+10.8 \\
+61.1\end{array}$ & $\begin{array}{l}1.43 \\
1.89\end{array}$ & $\begin{array}{l}-0.58 \\
-0.50\end{array}$ & $\begin{array}{l}-0.16 \\
+0.12\end{array}$ & $\begin{array}{l}+0.10 \\
+0.33\end{array}$ \\
\hline $30 \mathrm{~A}$ & 0 to 8 & Carbohydrate $193^{*}$ & 6.3 & 18.7 & 1.4 & 285 & & -37.8 & & 1.16 & -1.05 & -0.38 & \\
\hline 31 & 0 to 7 & Carbohydrate $171^{*}$ & 5.5 & 11.2 & 3.1 & 225 & & -22.3 & & 1.19 & -1.02 & -0.41 & \\
\hline $35 B$ & 0 to 8 & Carbohydrate $183^{*}$ & 5.5 & 13.2 & & 390 & & -37.2 & & 1.11 & -1.06 & -0.46 & \\
\hline $23 \mathrm{~L}$ & 0 to 9 & Carbohydrate $564^{*}$ & 17.7 & 28.0 & & 440 & & -77.0 & & 2.20 & -1.82 & -0.78 & \\
\hline $37 \mathrm{G}$ & 0 to 9 & Carbohydrate $565^{*}$ & 16.6 & 28.4 & & 1025 & & -105.9 & & 1.49 & -1.93 & -1.00 & \\
\hline $23 \mathrm{M}$ & 0 to 9 & Carbohydrate $562^{*}$ & 0 & 11.0 & & 310 & & -110.1 & & 1.27 & -1.05 & -1.10 & \\
\hline $47 \mathrm{~A}$ & 0 to 6 & Carbohydrate $360^{*}$ & 0 & 11.1 & & 305 & & -25.9 & & 0.96 & -0.89 & -0.37 & \\
\hline $49 A$ & 0 to 9 & Carbohydrate $539^{*}$ & 0 & 28.8 & & 685 & & -85.2 & & 2.20 & -2.11 & -0.67 & \\
\hline
\end{tabular}

* Received dry carbohydrate in amounts noted plus protein as gelatin capsules.

** $\Delta \mathrm{I}_{\mathrm{I}}$ omitted, since it is simply $\Delta \mathrm{W}-\Delta \mathrm{E}$.

probable that this animal had latent renal insufficiency, not evident prior to the experiment. These studies indicate that the high nitrogen concentrations found in urine during water deprivation are not maximal, and that they can be increased by the administration of protein.
However, this ability to increase nitrogen excretion without further water loss is limited, since the urine volume invariably increased with a higher daily metabolism of protein.

The concentration of non-protein nitrogen in the blood is an indirect measure of renal activity. 


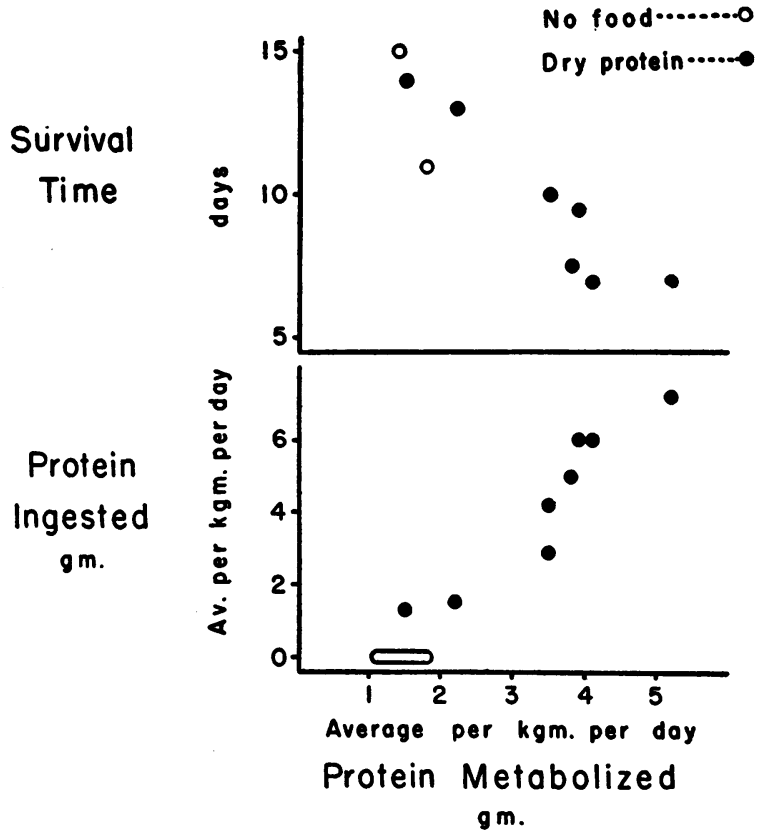

Fig. 1. Protein Metabolized is Charted Against Protein Ingested and Against Survival Time

As the metabolism of protein increased, the survival time decreased. After a survival period of 10 to 20 days, all but 2 of the control animals were resuscitated and hence do not appear in the upper graph. In the tables, periods of only 7 to 12 days are used for these animals for comparison with the feeding experiments.

In Figure 3, all values of blood non-protein nitrogen in the protein-fed dogs are plotted against time. The response was variable, since the non-protein nitrogen rose early in some and late in others. Apparently, in the majority, renal function was well maintained in spite of severe dehydration.

(D) Distribution of water loss. Control animals, as well as those maintained on dry protein, dry carbohydrate, or both, lost water from both phases. In percentage of initial volume, extracellular loss preponderated in about half of the animals; the intracellular loss was greater in the other half.

There is only a partial correlation between decline in the volume of extracellular fluid and an elevation in blood non-protein nitrogen. The extracellular fluid volume decreased early, and preponderated, in 3 of the 4 experiments with an early rise in blood non-protein nitrogen. In 3 of the 4 experiments with a late increase in the non-protein nitrogen, the loss from the intracellular phase was larger. These results suggest that a decrease in extracellular fluid volume affects renal circulation adversely.

The water loss in the dogs which received urea was distributed over both phases. The animals which were fed fish appear to have lost only intracellular water. There is no ready explanation for this unusual finding.

(E) Clinical course. Although the control animals lived longer than did the animals fed protein, the clinical course was similar. An accelerated weight loss was the only difference detected in the animals on protein ration. Vomiting and diarrhea were absent. The protein-fed animals continued to have formed stools, while the controls did not. The animals of both series tended to shiver, became very weak, and finally unresponsive.

\section{DISCUSSION}

Animals deprived of food and water rapidly become dehydrated, losing 27 to 36 per cent of their initial body water in 2 to 3 weeks (Table IB). Dehydration develops even in starving animals permitted free access to water, but it is not as severe (Table IB). Protein fed in excess of 1.5 grams per kilogram per day stimulates protein metabolism, accelerates water loss, and shortens the period of survival (Figure 1). Ingestion of smaller amounts of protein fails to increase protein metabolism (Figure 1), and hence does not accelerate water loss. The deleterious action of protein ingestion on water balance can be related to an increased urine volume consequent upon an increased formation of urea. The demand for the excretion of the additional urea is so imperative that water is sacrificed, even by the severely dehydrated organism. The organism does not conserve water by retention of urea, even though retention of water is a critical necessity. The increase observed in blood non-protein nitrogen was never sufficient to account for more than a fraction of the nitrogen metabolized.

Increase in the insensible loss of weight may sometimes contribute to the accelerated water loss following protein ingestion. It is reasonable to ascribe this to an increase in total metabolism, secondary to the increased protein metabolism. 


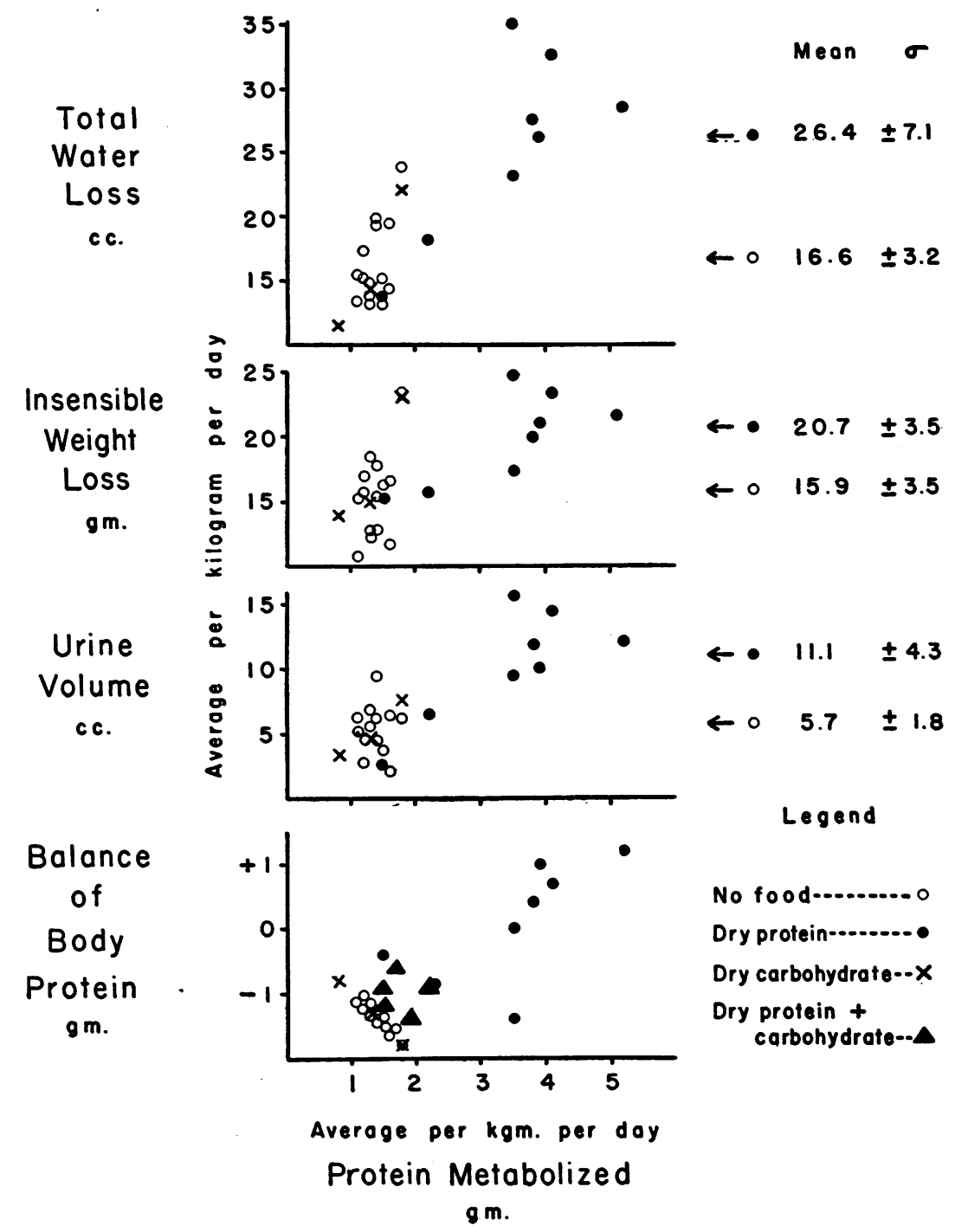

Fig. 2. Protein Metabolized is Charted Against Total Water Loss, Insensible Weight Loss, Urine Volume, and Balance of Body Protein, Expressed as AverAGES PER KILOGRAM PER DAY

The animals fed dry protein plus carbohydrate are only plotted in the lowest figure since they overlie the control groups. It is evident that dry protein may spare body protein but accelerates dehydration. In dogs, carbohydrate ingestion diminishes dehydration chiefly by sparing body protein.

There is evidence, however, that the state of hydration plays a significant rôle as well in determining the amount of weight lost insensibly. Thus, the insensible loss of weight in dogs fasted without water was, on the average, 20 per cent lower than in dogs fasted with water. This difference is correlated with a greater water loss in the animals deprived of food and water. It seems quite as likely that this difference results from an alteration in the usual relationship between insensible loss and total metabolism with severe dehydration, as that total metabolism itself is altered in dehydration.

Since insensible loss of weight is used in calculation of total calories and in turn in the estimation of fat burned and of water lost, an alteration 


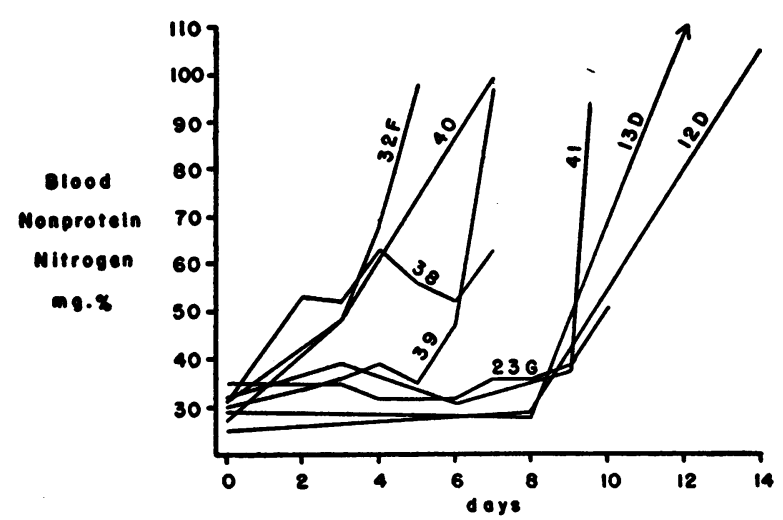

Fig. 3. Blood Non-Protein Nitrogen Concentrations in the Dogs Fed Dry Protein

Each line is based upon periods in individual experiments. The numbers of the experiments are identical with those in Table II. In the table, however, some of the intermediate periods have been omitted for the sake of economy.

in the usual relationship between total metabolism and insensible loss would affect the calculation of water balance. The alternative method for calculation of total calories independent of insensible loss, based on the calorimetric experiments of Anderson and Lusk (5), did result in higher values. In terms of water balance, the difference was too small to be significant.

These experiments clearly indicate that the water formed from the metabolism of protein together with that formed from the small amount of fat in the ration is insufficient to supply the extra water needed for the renal excretion of nitrogenous end-products. They also indicate that the dog cannot excrete the large amounts of extra urea without increasing the urine volume. Hence, the ingestion of protein food is advisable only if adequate amounts of water are available. As soon as water was administered at the close of Experiment 23G, the moribund animal recovered promptly, although the ingestion of protein was continued. Dogs remained in excellent condition, save for a slight weight loss, on a high protein intake of raw fish with no other source of water. Dogs placed on a similar intake of dry protein died even more rapidly than did those without any food or water. Therefore, only amounts of dry protein which do not increase protein metabolism above that of the fasting animal can be tolerated. In dogs, carbohydrate ingestion can benefit water balance by making available water of oxidation, and especially by sparing body protein. This reduces the urine volume by decreasing the amount of nitrogen requiring excretion. It is not possible to answer the general query as to whether carbohydrate can conserve body water by amelioration of ketosis, since marked ketosis does not develop in these animals (6).

These results with protein administration to dogs without water are applicable to human subjects (7). Since man cannot concentrate nitrogen to as great a degree as the dog (8), an even greater loss of water following the ingestion of dry protein might be expected.

\section{SUMMARY AND CONCLUSIONS}

(1) Dry protein in small amounts (1.5 grams per kilogram) without water maintains dogs in nitrogen equilibrium without change in the nitrogen excretion.

(2) Larger amounts of protein (2.0 to 7.2 grams per kilogram) increase the metabolism of protein and the nitrogenous end-products in the urine.

(3) The increased excretion of nitrogen necessitates a larger urine volume, even when dehydration is present.

(4) Dry protein in amounts greater than that necessary for nitrogen equilibrium, without adequate supplies of water, increases dehydration and decreases survival time.

(5) Similar amounts of protein with an adequate intake of water (whole fish) will maintain dogs in an excellent condition for at least 4 weeks.

(6) In the dog, administration of carbohydrate can conserve water. The economy of body water results chiefly from a decrease in protein metabolism and in the amount of nitrogen necessitating excretion. In addition, the water of oxidation of the carbohydrate is made available.

\section{BIBLIOGRAPHY}

1. Elkinton, J. R., and Taffel, M., Prolonged water deprivation in the dog. J. Clin. Invest., 1942, 21, 787.

2. Winkler, A. W., Elkinton, J. R., Hopper, J., Jr., and Hoff, H. E., Experimental hypertonicity: alterations in the distribution of body water, and the cause of death. J. Clin. Invest., 1944, 23, 103. 
3. Hopper, J., Jr., Elkinton, J. R., and Winkler, A. W., Plasma volume in dogs in dehydration with and without salt loss. J. Clin. Invest., 1944, 23, 111.

4. Straub, W., Ueber den Einfluss der Wasserentziehung auf den Stoffwechsel und Kreislauf. Ztschr. f. Biol., 1899, 38, 537.

5. Lusk, G., The Elements of the Science of Nutrition. W. B. Saunders Co., Philadelphia, 1928.

6. Crandall, L. A., Jr., A comparison of ketosis in man and dog. J. Biol. Chem., 1941, 138, 123.
7. Winkler, A. W., Danowski, T. S., Elkinton, J. R., and Peters, J. P., Electrolyte and fluid studies during water deprivation and starvation in human subjects, and the effect of ingestion of fish, of carbohydrate, and of salt solutions. J. Clin. Invest., 1944, 23, 807.

8. Hayman, J. M., Jr., Shumway, N. P., Dumke, P., and Miller, M., Experimental hyposthenuria. J. Clin. Invest., 1939, 18, 195. 